\title{
Optical parametric generation in orientation-patterned gallium phosphide
}

\author{
hanyu Ye, ${ }^{1}$ S. Chaitanya Kumar, ${ }^{2,}{ }^{*}$ Junxiong Wei, ${ }^{1}$ P. G. Schunemann, ${ }^{3}$ \\ M. EBRAHIM-ZADEH ${ }^{1,2,4}$ \\ ${ }^{1}$ ICFO-Institut de Ciencies Fotoniques, The Barcelona Institute of Science and Technology, 08860 Castelldefels (Barcelona), Spain \\ ${ }^{2}$ Radiantis, Polígon Camí Ral, 08850 Gavà, Barcelona, Spain \\ ${ }^{3}$ BAE Systems, Incorporated, MER15-1813, P.O. Box 868, Nashua, New Hampshire 03061-0868, USA \\ 4 Institucio Catalana de Recerca i Estudis Avancats (ICREA), Passeig Lluis Companys 23, 08010 Barcelona, Spain \\ *Corresponding author: chaitanya.suddapalli@radiantis.com
}

Received XX Month XXXX; revised XX Month, XXXX; accepted XX Month XXXX; posted XX Month XXXX (Doc. ID XXXXX); published XX Month XXXX

\begin{abstract}
We report the first optical parametric generator (OPG) based on the new nonlinear material, orientationpatterned gallium phosphide (OP-GaP). Pumped by a Qswitched nanosecond Nd:YAG laser at $1064 \mathrm{~nm}$ with 25 $\mathrm{kHz}$ pulse repetition rate, the OPG can be tuned across $1721-1850 \mathrm{~nm}$ in the signal and $2504-2787 \mathrm{~nm}$ in the idler. Using a 40-mm-long crystal in single pass, we have generated a total average output power of up to $\sim 18 \mathrm{~mW}$, with $\sim 5 \mathrm{~mW}$ of idler power at $2670 \mathrm{~nm}$, for $2 \mathrm{~W}$ of input pump power. The OPG exhibits a passive stability in total output power better than $0.87 \%$ rms over 1 hour, at a crystal temperature of $120{ }^{\circ} \mathrm{C}$, compared to $0.14 \%$ rms for the input pump. The output signal pulses, recorded at $1769 \mathrm{~nm}$, have duration of $5.9 \mathrm{~ns}$ for input pump pulses of $9 \mathrm{~ns}$. Temperature-dependent loss measurements for the pump polarization along the $[100]$ axis in the OP-GaP crystal have also been performed, for the first time, indicating a drop in transmission from $28.8 \%$ at 50 으 $\mathrm{C}$ to $19.4 \%$ at $160^{\circ} \mathrm{C}$. (C) 2015 Optical Society of America
\end{abstract}

OCIS codes: (190.4360) Nonlinear optics, devices; (190.4400) Nonlinear optics, materials; (190.4410) Nonlinear optics, parametric processes.

http://dx.doi.org/10.1364/OL.99.099999

Mid-infrared (mid-IR) coherent sources based on optical parametric down-conversion are of great significance for a variety of applications such as spectroscopy [1], up-conversion imaging [2] and as pump source for other nonlinear processes [3]. Oxide-based nonlinear crystals, and especially their quasi-phase matched (QPM) counterparts such as MgO-doped periodically-poled $\mathrm{LiNbO}_{3}$ (MgO:PPLN), stoichiometric $\mathrm{LiTaO}_{3}$ (MgO:sPPLT) and $\mathrm{KTiOPO}_{4}$ (PPKTP), have enabled broadband mid-IR coverage up to $\sim 4 \mu \mathrm{m}$, in all time-scales from continuous-wave (CW) to femtosecond domain [4-7]. However, the onset of multiphonon absorption in these nonlinear crystals has been a fundamental barrier to reach spectral regions into the deep mid-IR. Non-oxide-based nonlinear materials with extended transparency in the mid-IR, such as $\mathrm{ZnGeP}_{2}$ (ZGP) and orientation-patterned GaAs (OP-GaAs), offer potential for the development of parametric sources beyond $4 \mu \mathrm{m}$. However, their low bandgap requires pumping beyond $2 \mu \mathrm{m}$ to avoid two-photon absorption. The more recently developed nonlinear crystal, $\mathrm{CdSiP}_{2}$ (CSP), is an important new addition to the family of birefringent nonlinear materials for parametric downconversion into the deep mid-IR. The high optical nonlinearity, noncritical phase-matching (NCPM) capability, and a wide transparency range extending down to below $\sim 1 \mu \mathrm{m}$, enable the deployment of the widely available Nd-based solid-state or Ybbased fiber lasers as pump source for deep mid-IR wavelength generation up to $\sim 7 \mu \mathrm{m}[8,9]$. On the other hand, the new semiconductor nonlinear material, orientation-patterned GaP (OP$\mathrm{GaP}$ ) can be considered as the QPM analog of CSP, and a promising alternative for deep mid-IR generation. As a QPM material, OP-GaP offers unique flexibility for tunable wavelength generation into the deep mid-IR using grating engineering under NCPM with no spatial walk-off. Moreover, unlike ZGP and OP-GaAs, it has significantly lower two-photon absorption, allowing the direct use of well-established laser pump sources at $\sim 1 \mu \mathrm{m}$ for parametric down-conversion. Together with a wide transparency up to $\sim 12$ $\mu \mathrm{m}$, large optical nonlinearity $\left(d_{14}=70.6 \mathrm{pm} / \mathrm{V}\right)$ and high thermal conductivity $(110 \mathrm{~W} / \mathrm{mK})$ [10], these characteristics make OP-GaP a highly attractive alternative for the development of practical parametric sources throughout the mid- to deep-IR.

Earlier reports on nanosecond optical parametric sources based on OP-GaP include nanosecond doubly-resonant optical parametric oscillator (DRO) pumped at a $1064 \mathrm{~nm}$, generating 4 $\mathrm{mW}$ of idler at $4624 \mathrm{~nm}$ and $15 \mathrm{~mW}$ of signal at $1324 \mathrm{~nm}$ at $10 \mathrm{kHz}$ [11], as well as a nanosecond DRO pumped at $2090 \mathrm{~nm}$, operating at an idler wavelength of $5100 \mathrm{~nm}$ and a signal wavelength of 3540 $\mathrm{nm}$, with a total signal plus idler average output power of $350 \mathrm{~mW}$ at $20 \mathrm{kHz}$ repetition rate [12]. We also recently demonstrated tunable difference-frequency-generation (DFG) source based on 
OP-GaP by mixing the input pulses from a nanosecond Nd:YAG pump laser and the signal from a MgO:PPLN OPO in a 40-mm-long crystal, resulting in the generation of tunable mid-IR radiation over $2548-2781 \mathrm{~nm}$ with $\sim 14 \mathrm{~mW}$ of output power at $2719 \mathrm{~nm}$ at 80 $\mathrm{kHz}$ repetition rate [13]. In the CW regime, single-pass DFG based on 16.5-mm-long OP-GaP crystal was reported, providing up to $150 \mathrm{~mW}$ at $3400 \mathrm{~nm}$ for an input pump power of $47 \mathrm{~W}$ at $1064 \mathrm{~nm}$ together with $24 \mathrm{~W}$ of signal power at $1550 \mathrm{~nm}$ [14]. Recently, using a $24.6 \mathrm{~mm}$-long-OP-GaP crystal, a CW DFG power of $65 \mu \mathrm{W}$ was generated at $5850 \mathrm{~nm}$ for a pump power of $10 \mathrm{~W}$ at $1064 \mathrm{~nm}$ and a signal power of $40 \mathrm{~mW}$ at $1301 \mathrm{~nm}$ [15].

In this letter, we report what we believe to be the first optical parametric generator (OPG) based on OP-GaP. The OPG, pumped by a Q-switched Nd:YAG laser at $1064 \mathrm{~nm}$, can be temperature tuned across $1721-1850 \mathrm{~nm}$ in the signal and 2504-2787 $\mathrm{nm}$ in the idler, providing up to $\sim 18 \mathrm{~mW}$ of the total average output power at $25 \mathrm{kHz}$ repetition rate, with $\sim 5 \mathrm{~mW}$ of the idler at 2670 $\mathrm{nm}$. We have also performed systematic measurements of transmission at $1064 \mathrm{~nm}$ for polarization along the [100] axis in the OP-GaP sample, revealing a dramatic decrease in pump transmission with increase in crystal temperature.

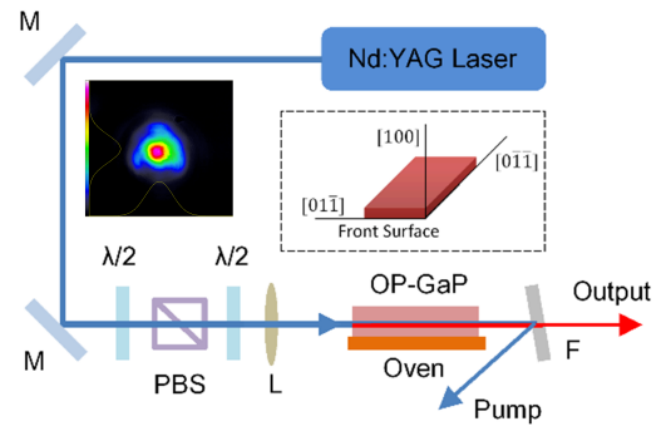

Fig. 1. Schematic of the experimental setup for the OP-GaP OPG. $\lambda / 2$, half-wave plate; PBS, polarizing beam splitter; L, lens; F, filter; Inset: Spatial beam profile of the pump.

The schematic of the experimental setup for the OPG is shown in Fig. 1. The pump laser is a linearly polarized, Q-switched Nd:YAG laser (Bright Solutions, Sol) delivering up to $\sim 30 \mathrm{~W}$ of average power with a tunable repetition rate ranging from 20 to $100 \mathrm{kHz}$. It operates at a central wavelength of $1064 \mathrm{~nm}$ with a full-width at half-maximum (FWHM) bandwidth of $<0.5 \mathrm{~nm}$ and exhibits spectral jitter of $\sim 1 \mathrm{~nm}$ over 30 seconds. For pumping the OPG, we chose a fixed repetition rate of $25 \mathrm{kHz}$ in order to obtain higher peak pulse power for increased nonlinear conversion efficiency. Also shown in the inset of Fig. 1 is the spatial profile of the pump beam, together with orthogonal intensity profiles, indicating a single-peak Gaussian intensity distribution with a circularity $85 \%$. The pump power is controlled by the combination of a half-wave plate and a polarizing beam-splitter (PBS). The polarization of the pump is initially set along [100] for OP-GaP, as described in [14], and further adjusted using a second half-wave plate to achieve maximum efficiency for parametric generation in the crystal. The OP-GaP crystal is 40-mm-long, 6-mm-wide, 1.7-mm-thick, with a single grating period of $\Lambda=15.5 \mu \mathrm{m}$, and is mounted on an oven providing temperature control from room temperature to $200{ }^{\circ} \mathrm{C}$, with an accuracy of $0.1{ }^{\circ} \mathrm{C}$. The end faces of the crystal are antireflection (AR)-coated $(R<1 \%)$ for $1064 \mathrm{~nm}$ and 1500-1900 $\mathrm{nm}$, with high transmission $(R<20 \%)$ over $2500-2700 \mathrm{~nm}$. The pump beam is focused to a beam waist radius of $w_{0} \sim 89 \mu \mathrm{m}$ in the QPM layer of the crystal, corresponding to a focusing parameter of $\xi \sim 0.28$. A long-pass filter transmitting above $1.65 \mu \mathrm{m}$ is used to separate the total output power (signal plus idler) from the pump. Another long-pass filter transmitting above $2.4 \mu \mathrm{m}$ is used to extract only the idler power.

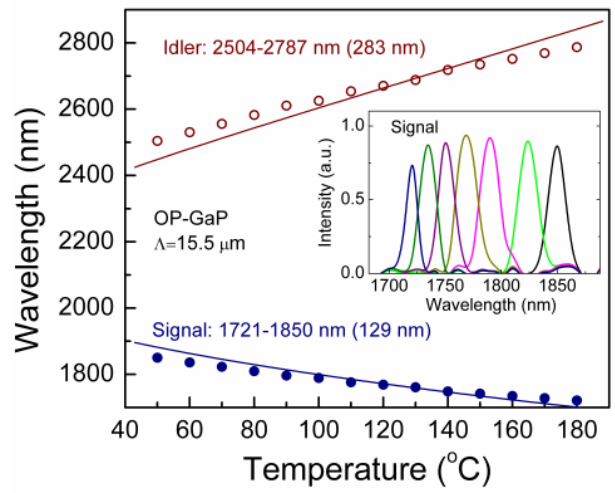

Fig. 2. Wavelength tuning range of the OP-GaP OPG for a grating period of $\Lambda=15.5 \mu \mathrm{m}$. Circles represent experimental measurements and the solid lines represent theoretical calculation. Inset: spectrum of the signal across tuning range.

Initially, we investigated the temperature tuning characteristics of the OPG. By changing the temperature of the OP-GaP crystal over $50-180{ }^{\circ} \mathrm{C}$, we were able to tune the generated signal across $1850-1721 \mathrm{~nm}$, together with the corresponding idler over 2504$2787 \mathrm{~nm}$, resulting in a total (signal plus idler) tuning over $412 \mathrm{~nm}$. The results are shown in Fig. 2, where the solid circles represent the experimentally measured signal wavelengths, while the hollow circles are the corresponding idler wavelengths estimated from energy conservation. The solid curves are the theoretical signal and idler wavelengths calculated using the relevant Sellmeier equations [11]. The temperature tuning curves indicate a wavelength tuning rate of $\sim 1 \mathrm{~nm} /{ }^{\circ} \mathrm{C}$ for the signal and $\sim 2.2 \mathrm{~nm} /{ }^{\circ} \mathrm{C}$ for the idler. Also shown in the inset of Fig. 2 is the signal spectra across the OPG tuning range, measured using a spectrometer with low resolution of $\sim 11 \mathrm{~nm}$.

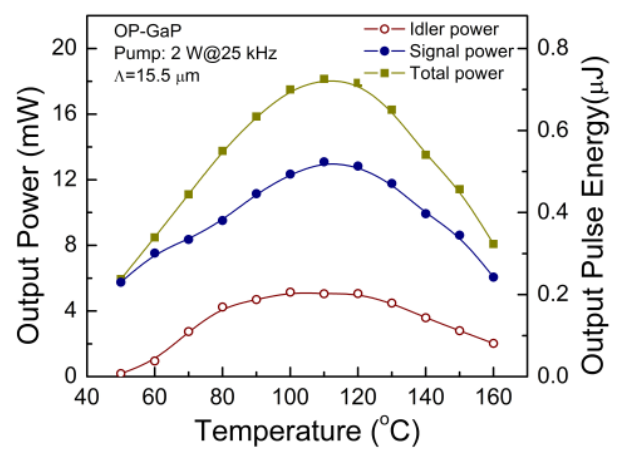

Fig. 3. Power and energy across the OPG tuning range as a function of the OP-GaP crystal temperature. 
The signal, idler and total output powers, and the corresponding pulse energies across tuning range of the OPG as a function of crystal temperature are shown in Fig. 3. As can be seen, the output power from the OPG follows a bell shaped variation as a function of temperature. For a fixed pump power of $2 \mathrm{~W}$ (corresponding to a pulse energy of $80 \mu \mathrm{J})$, the total OPG output power increases from $5.9 \mathrm{~mW}(0.24 \mu)$ to $18 \mathrm{~mW}(0.72 \mu))$ as the temperature of the OP$\mathrm{GaP}$ crystal is increased from $50^{\circ} \mathrm{C}$ to $110^{\circ} \mathrm{C}$. However, further increasing the temperature up to $160{ }^{\circ} \mathrm{C}$ results in the decline in output power down to $8.1 \mathrm{~mW}(0.32 \mu \mathrm{J})$. The recorded maximum total OPG output power of $18 \mathrm{~mW}(0.72 \mu \mathrm{J})$ at $110{ }^{\circ} \mathrm{C}$ corresponds to a measured idler power of $5 \mathrm{~mW}(0.2 \mu)$ at $2654 \mathrm{~nm}$ and an estimated signal power of $13 \mathrm{~mW}(0.52 \mu \mathrm{J})$ at $1776 \mathrm{~nm}$. The filtered idler power varies from $0.2 \mathrm{~mW}$ ( $8 \mathrm{~nJ})$ to $2 \mathrm{~mW}(80 \mathrm{n})$ with a maximum of $5.1 \mathrm{~mW}(0.204 \mu \mathrm{J})$ at $120{ }^{\circ} \mathrm{C}$, while the estimated signal power follows the idler, varying from $5.8 \mathrm{~mW}$ $(0.23 \mu \mathrm{J})$ to $6.1 \mathrm{~mW}(0.24 \mu \mathrm{J})$ as the temperature of the OP-GaP crystal is increased from $50{ }^{\circ} \mathrm{C}$ to $160 \stackrel{\circ}{\circ} \mathrm{C}$. Considering the measured transmission of the pump at $110^{\circ} \mathrm{C}$, the generated 18 $\mathrm{mW}$ of output power from OPG represents a single-pass conversion efficiency of $3.7 \%$ and photon conversion efficiency of $4.5 \%, 2.6 \%$ for the signal and idler, respectively. While varying the temperature of the OP-GaP crystal, we noticed that the position of the transmitted pump beam through sample, monitored using a scanning beam profiler, significantly shifted vertically, leading to the deviation of the pump beam from the thin QPM region. We attribute the drop in output power on either side of the maximum to crystal absorption at the pump, signal and/or idler wavelengths, as well as the observed deviation in the pump beam propagation direction out of the QPM grating. As such, we further studied the transmission characteristics of the crystal at the pump wavelength of $1064 \mathrm{~nm}$ for polarization set along the [100] direction.

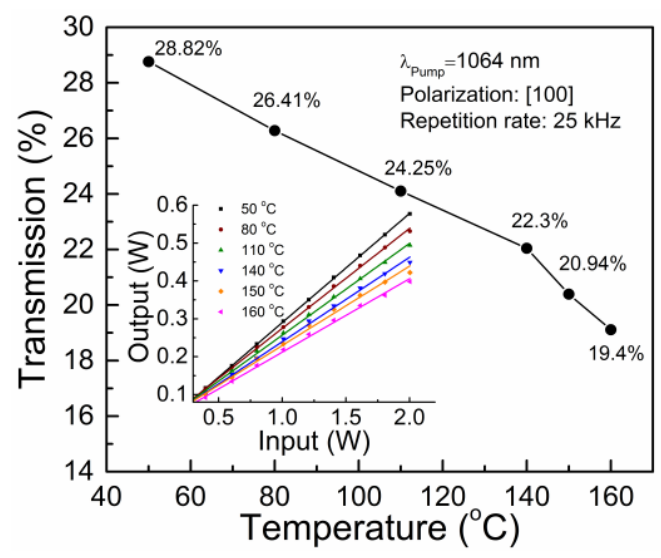

Fig. 4. Variation of the averaged transmission of the OP-GaP crystal at $1064 \mathrm{~nm}$ as a function of temperature. Inset: Transmitted pump power versus input pump power at different temperatures.

To obtain reliable data, we measured the transmitted pump power through the OP-GaP crystal as a function of the input pump power varying from $0.4 \mathrm{~W}(16 \mu \mathrm{J})$ to $2 \mathrm{~W}(80 \mu \mathrm{J})$, at six different temperatures, as shown in the inset of Fig. 4. In a separate measurement, we also estimated the damage threshold of the OPGaP crystal at $1064 \mathrm{~nm}$ to be $\sim 0.8 \mathrm{~J} / \mathrm{cm}^{2}$ when operating close to room temperature. In order to avoid any damage to the crystal, we thus limited the average pump power in our experiments to $2 \mathrm{~W}$ at
$25 \mathrm{kHz}$, corresponding to an energy fluence of $0.32 \mathrm{~J} / \mathrm{cm}^{2}$, well below the damage threshold of the OP-GaP sample. As the photon conversion efficiency in the OPG is very low $(<5 \%)$, we consider the entire output power from the crystal measured without any filters as the transmitted pump power, and further treat the slope efficiency as averaged transmission at every temperature point. As can be seen in Fig. 4, the pump transmission is unexpectedly low, amounting only to $28.8 \%$ at a crystal temperature of $50 \stackrel{\circ}{\circ}$, and further decreasing down to $19.4 \%$ at $160 \stackrel{\circ}{\circ}$. We also attempted to measure the transmission at $170{ }^{\circ} \mathrm{C}$. However, we observed the sudden appearance of a damage spot on the surface of the crystal at an input pump power of $2 \mathrm{~W}(80 \mu \mathrm{J})$ at $170^{\circ} \mathrm{C}$, implying the OP$\mathrm{GaP}$ crystal has lower damage threshold at higher temperatures due to the stronger pump absorption. To the best of our knowledge, this is the first experimental investigation of temperature-dependent optical transmission in OP-GaP.

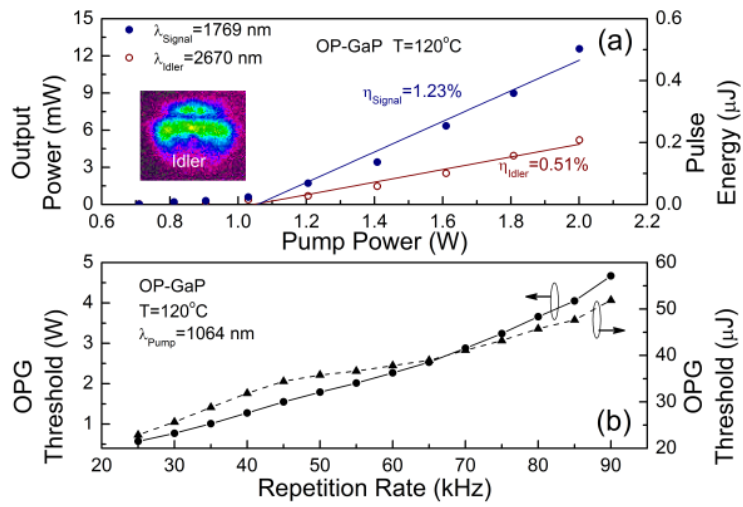

Fig. 5. (a) Signal and idler power scaling as a function of the pump power; (Inset) Idler output beam profile. (b) dependence of the OPG threshold on the pump laser repetition rate at an OP-GaP crystal temperature of $120^{\circ} \mathrm{C}$.

The power scaling characteristics of the generated signal and idler from the OPG measured at repetition rate of $25 \mathrm{kHz}$ and an OP-GaP crystal temperature of $120^{\circ} \mathrm{C}$ are presented in Fig. 5(a). For a maximum pump power of $2 \mathrm{~W}(80 \mu \mathrm{J})$, corresponding to a pump energy fluence of $0.32 \mathrm{~J} / \mathrm{cm}^{2}$ in the OP-GaP crystal, we were able to generate $12.6 \mathrm{~mW}(0.5 \mu)$ of signal power at $1769 \mathrm{~nm}$ together with $5.2 \mathrm{~mW}(0.21 \mu \mathrm{J})$ of idler power at $2670 \mathrm{~nm}$. The slope efficiencies are estimated to be $1.23 \%$ and $0.51 \%$ for the signal and idler, respectively. It is to be noted that the data presented here are not corrected for the $\sim 20 \%$ AR-coating loss of the OP-GaP crystal in the idler wavelength range. Further, we explored the OPG threshold at different repetition rates varying from $25 \mathrm{kHz}$ to $90 \mathrm{kHz}$. The threshold values were measured at a fixed OP-GaP crystal temperature of $120^{\circ} \mathrm{C}$ using a high-sensitivity visible spectrometer (Ocean Optics, HR4000). The criterion for OPG threshold measurement was the visual observation of red light resulting from parasitic sum-frequency-generation of pump and generated signal, which suddenly appeared as the pump power was gradually increased. The OPG threshold as a function of the pump pulse repetition rate is shown in Fig. 5(b). As can be seen, the threshold increases from $0.57 \mathrm{~W}(23 \mu)$ at $25 \mathrm{kHz}$ to $4.67 \mathrm{~W}$ $(52 \mu)$ at $90 \mathrm{kHz}$, due to the drop in the peak pump power at higher repetition rates, as expected. Although we were not able to measure the signal beam profile, due to lack of suitable beam 
separation filters, the OPG idler output beam profile is shown in the inset of Fig. 5. The beam has an elliptic double-lobe structure (circularity $>60 \%$ ), attributed to the low circularity of the pump beam, non-uniform grating aperture and inhomogeneous quality of the OP-GaP sample. With improved crystal quality and pump circularity, major enhancement in beam quality is expected.

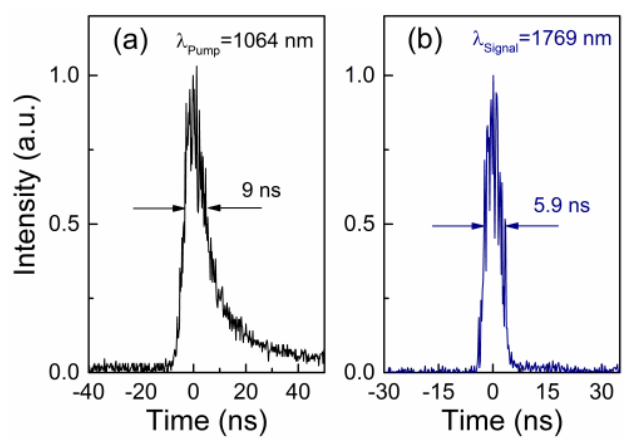

Fig. 6. Measured pulse shapes of the (a) pump, and (b) signal for the $\mathrm{OP}-\mathrm{GaP} \mathrm{OPG}$ at $25 \mathrm{kHz}$ repetition rate.

We measured the pulse duration of the pump and the generated signal from the OPG using an InGaAs detector with a bandwidth of $5 \mathrm{GHz}$ and a digital oscilloscope with a bandwidth of $3.5 \mathrm{GHz}$. As shown in Fig. 6(a,b), the typical FWHM pulse durations are recorded to be $9 \mathrm{~ns}$ for the pump at $1064 \mathrm{~nm}$ and $5.9 \mathrm{~ns}$ for the signal at a wavelength of $1769 \mathrm{~nm}$, at $25 \mathrm{kHz}$ repetition rate. It is also to be noted that closer inspection of the two temporal profiles reveals the beat frequency between different longitudinal modes in both pump and signal pulses.

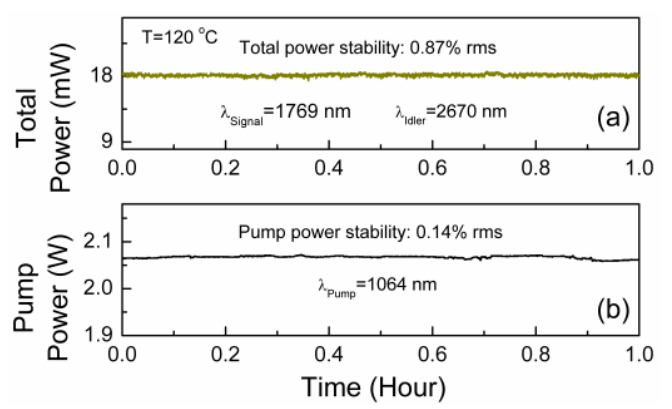

Fig. 7. Power stability of the (a) total output power from the OP-GaP $\mathrm{OPG}$, and (b) pump over a period of 1 hour.

Further, we investigated the long-term stability of the output power from the OPG. The power stability measurement was performed at an OP-GaP crystal temperature of $120{ }^{\circ} \mathrm{C}$, corresponding to the signal and idler wavelengths of $1769 \mathrm{~nm}$ and $2670 \mathrm{~nm}$, respectively. The results are shown in Fig. 7(a,b). The total output power from OPG exhibit a passive power stability better than $0.87 \%$ rms with a mean value of $18.15 \mathrm{~mW}$ over 1 hour, as compared to $0.14 \%$ rms with a mean value of $2.07 \mathrm{~W}$ for the simultaneously measured pump over the same period.

In conclusion, we demonstrated what is to our knowledge the first OPG based on OP-GaP. Using a nanosecond pulsed Nd:YAG laser at $25 \mathrm{kHz}$ repetition rate as the pump source and a $40-\mathrm{mm}$ - long OP-GaP crystal with a single grating period of $\Lambda=15.5 \mu \mathrm{m}$, we have achieved tunable single-pass parametric generation across the near- and mid-IR wavelength range of 1721-1850 nm in the signal and 2504-2787 $\mathrm{nm}$ in the idler, in close agreement with the theoretical calculation. The OPG can provide a total average output power of up to $\sim 18 \mathrm{~mW}(0.72 \mu \mathrm{J})$, with $\sim 5 \mathrm{~mW}(0.2 \mu \mathrm{J})$ of idler power at $2670 \mathrm{~nm}$, for a maximum pump power of $2 \mathrm{~W}(80 \mu \mathrm{J})$, with good passive power stability of $0.87 \%$ rms over 1 hour. The OPG output signal pulses have typical duration of 5.9 ns for input pump pulses of 9 ns. In addition, we have performed temperaturedependent transmission measurements for pump polarization along the [100] direction, revealing decreasing transmission of the OP-GaP crystal with increasing temperature. With further progress in the growth of OP-GaP crystals of higher optical quality and reduced transmission loss, substantial improvements in OPG efficiency, output power, and potential for power scaling are expected, paving the way for the development of practical parametric sources throughout the mid- to deep-IR using widely available solid-state and fiber laser pump sources near $1 \mu \mathrm{m}$.

We acknowledge support from Spanish Ministry of Economy and Competetiveness (MINECO) (nuOPO, TEC2015-68234-R); European Commission (Project Mid-Tech, H2020-MSCA-ITN2014); Agència de Gestió d'Ajuts Universitaris i de Recerca (AGAUR) (SGR 2014-2017); CERCA Programme / Generalitat de Catalunya, Severo Ochoa Programme for Centres of Excellence in R\&D (SEV-2015-0522); Fundació Privada Cellex.

Hanyu Ye acknowledges the support of Marie Curie Actions: Innovative Training Network through the Mid-Tech project (H2020-MSCA-ITN-2014).

\section{References}

1. T. Steinle, F. Neubrech, A. Steinmann, X. Yin, and H. Giessen, Opt. Express 23, 11105 (2015).

2. M. Mathez, P. J. Rodrigo, P. Tidemand-Lichtenberg, and C. Pedersen, Opt. Lett. 42, 579 (2017).

3. A. Sabella, J. A. Piper, and R. P. Mildren, Opt. Lett. 39, 4037 (2014).

4. S. Chaitanya Kumar and M. Ebrahim-Zadeh, Opt. Lett. 38, 5349 (2013).

5. S. Chaitanya Kumar, J. Wei, J. Debray, V. Kemlin, B. Boulanger, H. Ishizuki, T. Taira, and M. Ebrahim-Zadeh, Opt. Lett. 40, 3897 (2014).

6. S. Chaitanya Kumar, R. Das, G. K. Samanta, and M. Ebrahim-Zadeh, Appl. Phys. B 102, 31 (2011).

7. S. Chaitanya Kumar, A. Esteban-Martin, T. Ideguchi, M. Yan, S. Holzner, T. W. Hänsch, N. Picqué, and M. Ebrahim-Zadeh, Laser \& Photon. Rev. 8, L86 (2014).

8. S. Chaitanya Kumar, P. G. Schunemann, K. T. Zawilski, and M. EbrahimZadeh, J. Opt. Soc. Am. B 33, D44 (2016).

9. S. Chaitanya Kumar, A. Esteban-Martin, A. Santana, K. T. Zawilski, P. G. Schunemann, and M. Ebrahim-Zadeh, Opt. Lett. 41, 3355-3358 (2016).

10. P. G. Schunemann, K. T. Zawilski, L. A. Pomeranz, D. J. Creeden, and P. A. Budni, J. Opt. Soc. Am. B 33, D36 (2016).

11. L. A. Pomeranz, P. G. Schunemann, D. J. Magarrell, J. C. McCarthy, K. T. Zawilski, and D. E. Zelmon, in Conference on Lasers and Electro-Optics (CLEO) (Optical Society of America, 2015), paper SW30.4.

12. P. G. Schunemann, L. A. Pomeranz, and D. J. Magarrell, in Conference on Lasers and Electro-Optics (CLEO) (Optical Society of America, 2015), paper SW30.1.

13. J. Wei, S. Chaitanya Kumar, H. Ye, K. Devi, P. G. Schunemann, and M. Ebrahim-Zadeh, Opt. Lett. 42 (2017) (In press).

14. S. Guha, J. O. Barnes, and P. G. Schunemann, Opt. Mater. Express 5, 2911 (2015). 
15. G. Insero, C. Clivati, D. D’Ambrosio, P. De Natale, G. Santambrogio, P. G. Schunemann, J. J. Zondy, and S. Borri, Opt. Lett. 41, 5114 (2016). 